\title{
Superconducting detector dynamics studied by quantum pump-probe spectroscopy
}

\author{
Reinier W. Heeres and Valery Zwiller \\ Kavli Institute of Nanoscience, Delft University of Technology, P.O. Box 5046, 2600 GA Delft, \\ The Netherlands
}

(Received 25 July 2012; accepted 21 August 2012; published online 11 September 2012)

\begin{abstract}
We explore the dynamics of superconducting single-photon detectors (SSPDs) on the picosecond time-scale using a correlated photon-pair source based on spontaneous parametric downconversion (SPDC), corresponding to a pump-probe experiment at the single-photon level. We show that the detector can operate in a regime where the two-photon detection probability is orders of magnitude larger than the single-photon detection probability. The characteristic relaxation time-scale of the out-of-equilibrium hot-spot is found to be $\sim 15 \mathrm{ps}$. Our measurement technique is an effective tool to study fast two-photon processes, without requiring a power-dependence measurements to determine the number of photons involved. (C) 2012 American Institute of Physics.

[http://dx.doi.org/10.1063/1.4750139]
\end{abstract}

Superconducting single-photon detectors (SSPDs) ${ }^{1}$ are extremely useful photon detectors because of their broad operating wavelength range, small timing jitter, and short dead time, making them attractive candidates for integration in quantum communication and linear-optics based quantum computation schemes. ${ }^{2}$ It is important to better understand the dynamics ${ }^{3}$ of the detection process to further enhance the detection efficiency and to determine the fundamental limits in time-resolution and count-rate. The description of photondetection by an SSPD usually follows the so-called hot-spot model, ${ }^{4,5}$ in which a narrow nanowire carries a supercurrent close to the critical current. Absorbtion of a photon results in creation of an energetic quasi-particle. Electron-electron and electron-phonon interactions redistribute this energy and cause a growing hot-spot to form, expelling the supercurrent outwards. The electron-phonon interactions thermalize the electrons back to the substrate temperature. Both this process and details of the diffusion of the quasi-particles determine the relaxation time of the hotspot, $\tau_{R}$. If the energy of the photon and the initial bias current are large enough a normal, resistive region can be created over the whole width of the nanowire. This resistive constriction gives rise to a measurable voltage pulse, the shape of which is set by the kinetic inductance $L$, the hot-spot resistance, and the load impedance $R_{\text {load }}$. The latter results in a recovery time-scale $L / R_{\text {load }}{ }^{6}$ The hot-spot relaxation time $\tau_{R}$ is an important parameter, which we measure here for a regular detector using our single-photon pump-probe technique. The detector consists of a $100 \mathrm{~nm}$ wide nanowire meander with a $100 \mathrm{~nm}$ spacing patterned in a $\sim 4 \mathrm{~nm}$ thick $\mathrm{NbN}$ film on sapphire, covering a total area of about $2 \times 8 \mu \mathrm{m}$.

Pump-probe spectroscopy is a measurement technique to study fast (picosecond-scale or shorter) processes optically. Usually the response of a system is determined as a function of the time delay between the pump and probe pulse, generated by a pulsed laser diode or mode-locked laser. Since a spontaneous parametric downconversion (SPDC) source always produces photons in correlated pairs, ${ }^{7}$ one of the photons can take the place of the pump and the other of the probe pulse. The coherence time of such a source plays the role of the laser pulse length in traditional pumpprobe experiments and, therefore, sets the temporal resolution. This time-scale is determined by the thickness of the non-linear crystal involved and can be chosen from $\sim 100$ fs for thin crystals $(\sim 1 \mathrm{~mm})$ to $\sim 2$ ps for thick crystals $(\sim 2 \mathrm{~cm})$ as used here. In contrast to pump-probe experiments with laser pulses obeying Poissonian statistics, the photon number is well defined when using an SPDC source.

The first SSPD experiment ${ }^{1}$ already showed that lowering the applied bias current changes the power dependence of the detector. In particular, it goes from a linear power dependence at high bias to a quadratic or even higher order dependence at lower bias. ${ }^{8}$ This was interpreted as going from the regime where the detector is sensitive to a single photon to a regime where 2 or more photons are required to cause a detector click. It agrees well with theory, since the probability of having $N$ absorbed photons per pulse scales as $\sim p^{N}$ in the regime of pulsed optical excitation with an average photon number $p \ll 1$. The method we introduce here allows to directly probe the two-photon detection process without relying on the statistical photon-number distribution of Poissonian light through a power dependence measurement to determine the number of photons involved.

We start by estimating the probabilities of coincidental 2-photon and correlated 2-photon absorption events by a superconducting detector. Let $\eta_{a b s}$ denote the probability of photon absorption, including all system losses. Considering a Poissonian beam of average photon intensity $I$, the average number of absorbed photons within a time $\Delta t$ will be $\bar{n}=\eta_{a b s} I \Delta t$. The probability of absorbing $n$ photons within this time is given by the Poisson distribution $P(n ; \bar{n})$ $=\bar{n}^{n} e^{-\bar{n}} / n$ !. For events with 1 or 2 photons, this gives $P(1 ; \bar{n})=\bar{n} e^{-\bar{n}}$ and $P(2 ; \bar{n})=1 / 2 \bar{n}^{2} e^{-\bar{n}}$. The probability of a 2-photon absorption event when the photons come in pairs, however, is given by $P_{\text {pair }}=P(1 ; \bar{n}) \eta_{a b s}$, as the absorption of one photon heralds the presence of a second one. By comparing $P(2 ; \bar{n})$ and $P_{\text {pair }}(\bar{n})$, we conclude that when $\bar{n} \ll 2 \eta_{\text {abs }}$ the probability of absorbing a pair of correlated photons is 
much larger than an accidental 2-photon absorption event. The rates for 1-photon, coincidental 2-photon, and correlated 2-photon absorption events versus downconversion pair production rate are plotted in Fig. 1(a). The dashed vertical line indicates the pair generation rate in our setup, determined from the single and pair coincidence detection rates. ${ }^{9}$ The absorption efficiency $\eta_{a b s}$ is estimated to be $\sim 2 \times 10^{-3}$ (including all system losses), based on the count-rate at maximum bias current. The upper limit for $\Delta t$ to estimate $\bar{n}$ is taken to be $20 \mathrm{ps}$ from previous work on the lowest measured jitter $^{10}$ and traditional pump-probe measurements. ${ }^{11}$ Fig. 1(b) shows the ratio of pair-events versus total 2-photon events (coincidental plus correlated); indicating that in our operating regime, almost all 2-photon events are due to photons forming a pair; accidental two-photon events can be ignored. This is a crucial requirement for the applicability of our technique.

We model the "intrinsic detector efficiency," i.e., the probability to obtain a click when a photon is absorbed, as an activated process governed by an energy scale $E(I)$ that depends on the bias current. The probability of detection is given by $\eta_{d e t, \hbar \omega}=e^{-E(I) / \hbar \omega}$ for a 1-photon process and $\eta_{d e t, 2 \hbar \omega}=e^{-E(I) / 2 \hbar \omega}$ for a 2-photon process because this causes a disturbance with twice the energy. The overall count rate due to 1 -photon events is now given by $R_{1}=N \eta_{a b s}$ $e^{-E(I) / \hbar \omega}$ and for 2-photon events, $R_{2}=N \eta_{a b s}^{2} e^{-E(I) / 2 \hbar \omega}$. These functions are plotted in Figs. 1(d) and 1(e); line-cuts along the dotted lines are in Fig. 1(c). The requirement for observing pair events is that the single and pair count rates are of equal order of magnitude, which corresponds to the condition $e^{-E(I) / 2 \hbar \omega}=\eta_{a b s}$, i.e., the intrinsic pair detection efficiency $\eta_{d e t, 2 \hbar \omega}$ is equal to the absorption efficiency. For the single-photon detection efficiency, this means $\eta_{\text {det }, \text { hw }}$ $=\eta_{a b s}^{2}$. Increasing the energy scale $E(I)$ further by decreasing the bias current will improve the ratio of pair-detection versus single-detection efficiency as $e^{E(I) / 2 \hbar \omega}$ at the expense of a reduction of the absolute pair-detection efficiency by the same factor.

To include the effects of relaxation of the hotspot when two photons are separated by a time $d t$, we include a factor $e^{-d t^{2} / \tau_{R}^{2}}$ in our model, with $\tau_{R}$ the hotspot relaxation time. It is precisely this value that we can measure using our correlated photon pump-probe experiment. The two photons will also have to be absorbed at the same position in the nanowire, where the relevant length scale is the size of the hotspot created by a photon. This size is also governed by the thermalization process and is approximately given by the thermalization length $L_{t h}=\sqrt{D \tau_{t h}}$, with $D$ the carrier diffusion constant and $\tau_{\text {th }}$ the characteristic thermalization time. ${ }^{4}$ With typical values of $D \sim 0.5 \mathrm{~cm}^{2} \mathrm{~s}^{-1}$ and $\tau_{t h} \sim 20 \mathrm{ps}$, the expected hotspot radius is $\sim 30 \mathrm{~nm}$. The focused spot covers several hot-spot sizes, approximately $N_{s} \sim 80$ and, therefore the hotspots created by two photons have only a $\sim 1 / N_{s}$ probability of overlapping.

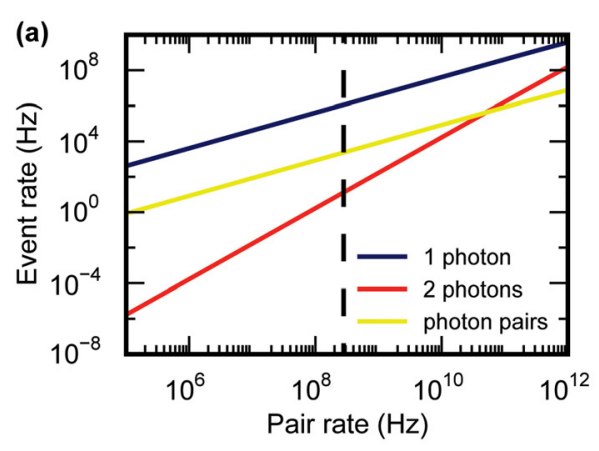

(d) $10^{-2}$

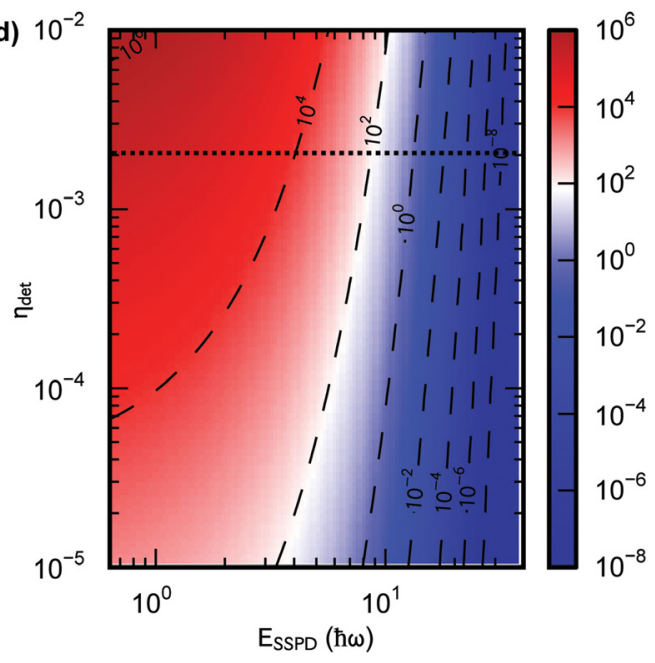

(b)

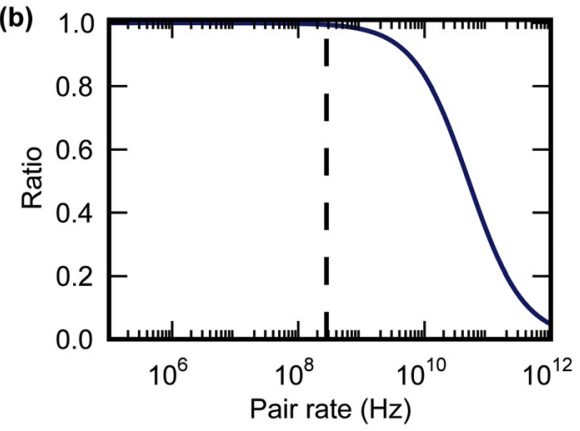

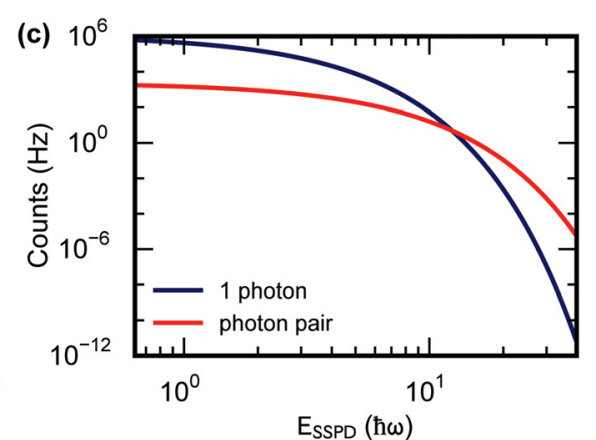

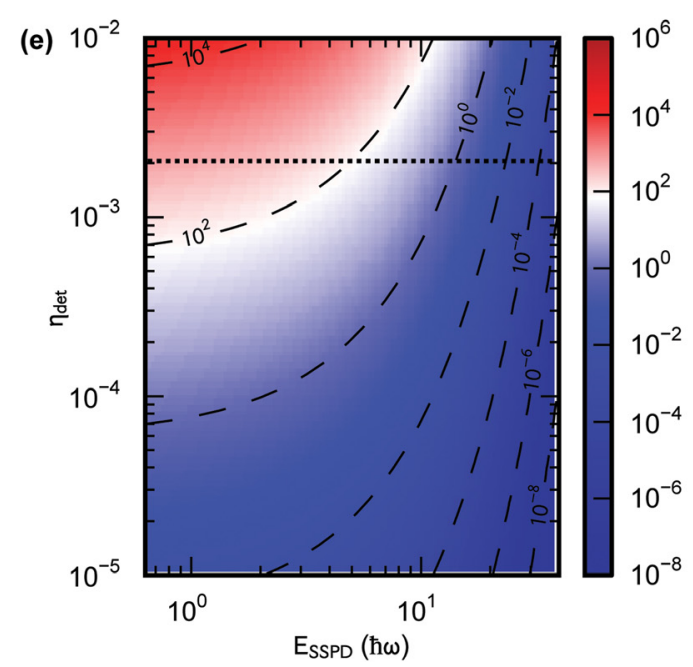

FIG. 1. (a) The number of 1-photon, accidental 2-photon, and correlated 2-photon absorption events as a function of the pair production rate with $\eta_{a b s}$ $=2 \times 10^{-3}$ and bin-size ( $\left.d t\right)$ of 20 ps. The dashed line indicates the pair production rate in this experiment. (b) The ratio of correlated 2 -photon events versus total (correlated and coincidental) 2-photon events as a function of pair production rate. (c) Count-rates caused by 1 photon events and by correlated 2-photon events as a function of SSPD energy scale in units of the single photon energy. (d) and (e) Single-photon (d) and 2-photon (e) count rates as a function of absorption probability $\eta_{a b s}$ and SSPD energy scale. Line-cuts along the dotted lines give (c). 
(a)

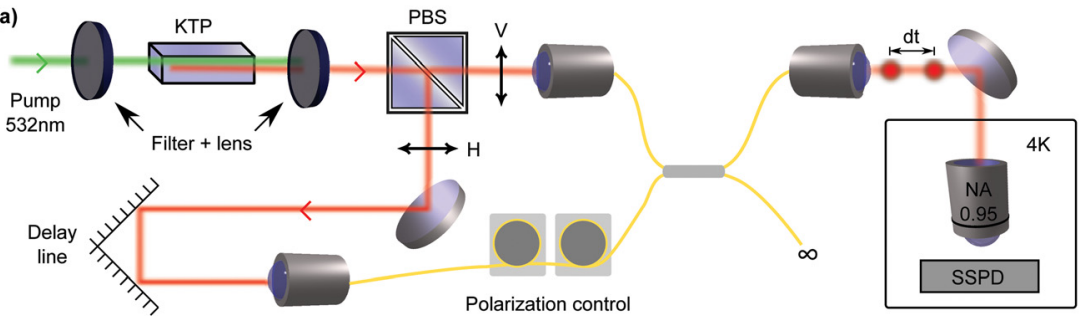

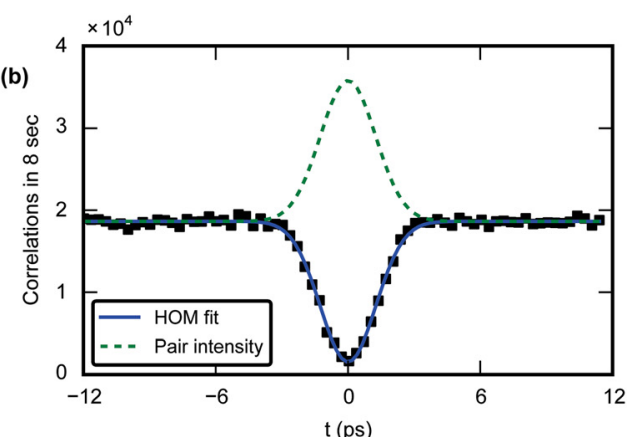

FIG. 2. (a) Experimental setup. Photon pairs are produced in a KTP crystal and separated on a polarizing beam splitter (PBS) after filtering out the pump beam. One of the photons is delayed with respect to the other in a motorized delay line. The photons are collected in single-mode fibers and combined using a fiber beam splitter. The output beam consists of photon pairs with a controlled delay dt and is focused to a $\sim 1 \mu \mathrm{m}$ spot on the SSPD using a high NA objective. (b) Photon pair source characterization by Hong-Ou-Mandel interference in the fiber beam splitter. The data points correspond to the number of coincidences between two APDs connected to both outputs of the fiber beam splitter (integration time $8 \mathrm{~s}$ ). If the photons arrive within the coherence time $\tau_{c}=2.96 \mathrm{ps}$, they bunch and travel as pairs to the same detector, causing the correlations to disappear. At the same time, the number of photon pairs in both arms increases and follows the green dashed line.

Our experimental setup is shown schematically in Fig. 2(a). The SPDC source is based on a $2 \mathrm{~cm}$ long non-linear potassium tytanyl phosphate (KTP) crystal cut for degenerate type-II phase-matching, on which we focus a $\sim 400 \mathrm{~mW}$, $532 \mathrm{~nm}$ continuous wave pump laser. After blocking the pump beam using a long-pass filter, we collect the correlated photons $(\lambda=1064 \mathrm{~nm})$ forming a pair in two separate optical fibers. Polarization control is performed in one of the fibers using stress-induced birefringence and the beams are combined again on a 50/50 fiber beam splitter. The time-delay $d t$ between the two photons can be controlled using a motorized delay line. If $d t$ is larger than the coherence time of the downconversion source, each of the photons individually exits into either of the two output fibers with $50 \%$ probability. In half of the cases, this will result in 1 photon in both outputs and in $25 \%$ of the cases, the upper arm contains 2 photons and the lower one zero. However, if the two photons arrive within the coherence time, Hong-Ou-Mandel (HOM) interference ${ }^{12}$ will cause the photons to bunch and leave in one of the outputs as a pair. Therefore, the cases where 1 photon is present in both output arms disappear. This is very clear in a correlation measurement between two avalanche photodiodes (APDs) connected to the outputs of the fiber beam splitter, and results in the characteristic HOM dip in Fig. 2(b), in this case with a coherence time $\tau_{c}=(2.96 \pm 0.04) \mathrm{ps}$. Simultaneously, the probability to get a photon pair in either arm doubles, so in 50\% of the cases, the upper output arm will contain a photon pair. This is indicated in Fig. 2(b) as the green dashed curve and has been demonstrated experimentally. ${ }^{13}$

The output of the photon pair source is focused to a diffraction limited spot of $\sim 1 \mu \mathrm{m}$ on a superconducting detector. Fig. 3(a) shows the measured number of detection events as a function of the time delay between the two photons forming a pair at several different bias currents. The broad peak at zero time delay is due to two-photon absorption events. The half-width of this peak corresponds directly to the hotspot relaxation time $\tau_{R}$. The narrower peak in the center is caused by the increased (at zero time-delay exactly doubled) number of photon pairs due to HOM interference. We fit the data with the function

$$
I(d t)=A+B e^{-d t^{2} / \tau_{R}^{2}} I_{\text {pairs }}(d t),
$$

where $\mathrm{A}$ is the background level, B the peak height, $\tau_{R}$ the $1 / e$ relaxation time, and $I_{\text {pairs }}(d t)$ the pair intensity as a result of HOM interference

$$
I_{\text {pairs }}(d t)=1+e^{-4 \ln (2) d t^{2} / \tau_{c}^{2}},
$$

with $\tau_{c}=(2.96 \pm 0.04) \mathrm{ps}$, the FWHM coherence time of the pair source, determined independently from Fig. 2(b).

The peak height and background level are plotted in Fig. 3(b). The ratio of the two (peak height/background) gives the signal to noise ratio (SNR) and is plotted as a solid line in the same graph. We find that, with increasing bias current, the background intensity grows much faster than the peak height and correspondingly that the SNR decreases. The fast relative increase of background intensity upon increasing the intrinsic detection efficiency indicates that the background is indeed caused by single-photon events and the peak by twophoton events.

Fig. 3(c) shows the same measurement at a fixed bias current and with different pumping powers of the downconversion source. The same fitting function is used and the resulting peak heights and backgrounds are plotted in Fig. 3(d), as well as their ratio. Here, we see that the background and peak height both increase approximately linearly as a function of pump power and, therefore, that the SNR stays constant. This is the expected behaviour since both the number of singles and the number of pairs scale linearly with pump power in a downconversion process. ${ }^{14}$ The $1 / e$ halfwidth of the broad peak is approximately $15 \mathrm{ps}$; it does not depend on the power and seems to be bias-independent within the experimental uncertainties. This is slightly lower than in previous work, ${ }^{11}$ possibly a result of the different substrate material used here.

We have demonstrated that two-photon detection using superconducting detectors is possible by applying a bias current of about $0.53 I_{c}$, much lower than the typical operating point for single-photon detection sensitivity. Our quantum pump-probe technique based on the use of correlated photons from a SPDC source has proven to be a straightforward tool and could be used to explore other two-photon effects without relying on statistics through power-dependence measurements to determine the number 
(a)
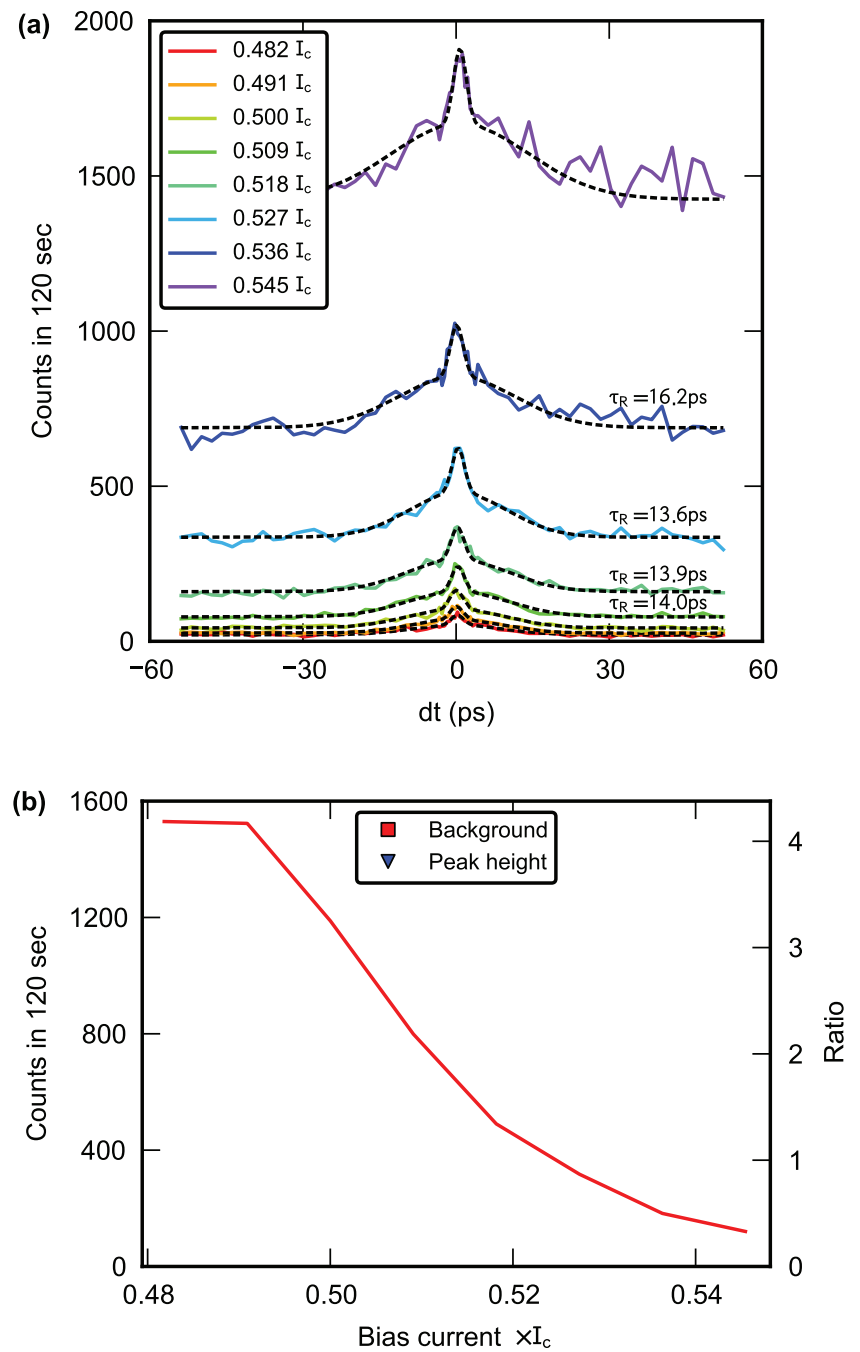

(c)

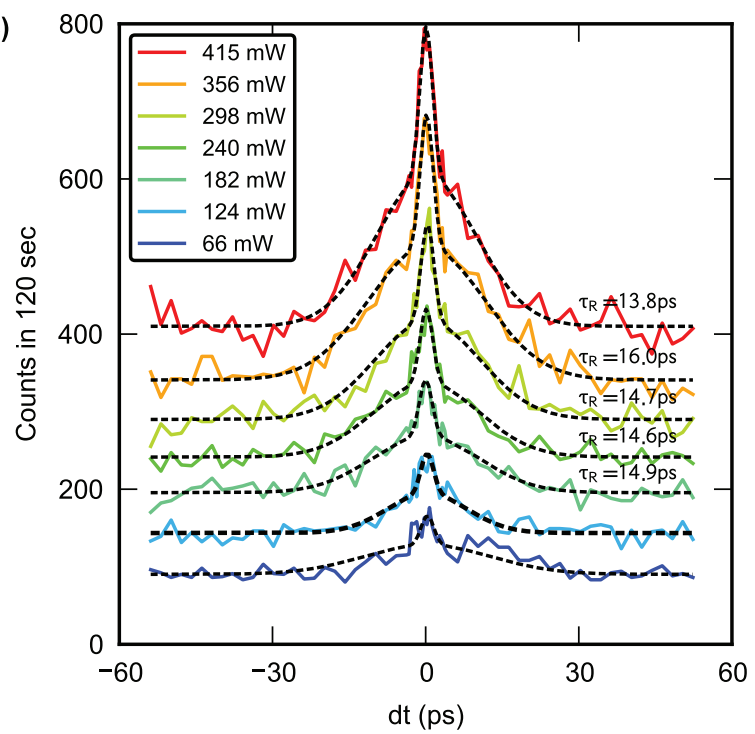

(d)

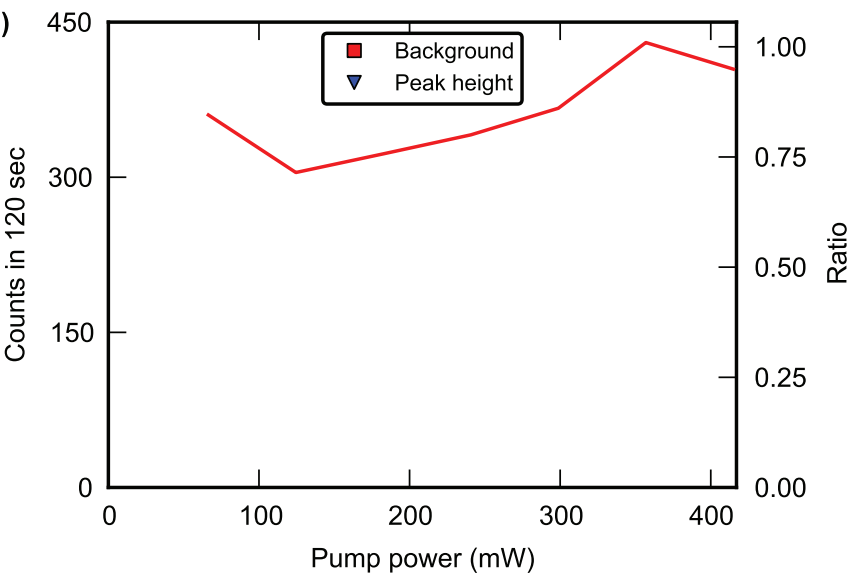

FIG. 3. (a) SSPD counts (in $120 \mathrm{~s}$ ) as a function of the delay between two photons forming a pair for several different bias currents. (b) Peak height and background level of the data in (a), indicating that at higher currents, the probability of detecting a single-photon (the background) grows faster than the probability of detecting a pair (the peak height). The solid line is the signal to noise ratio (peak height divided by background) using the right axis. (c) Same as (a), but for different SPDC pump powers. (d) Peak height and background level of the data in (c) and their ratio, showing that both the single- and pair-rates of the SPDC source depend linearly on the pump-power.

of photons involved. The hotspot relaxation time is found to be $\sim 15$ ps.

This work is supported financially by The Netherlands Organisation for Scientific Research (NWO/FOM).

${ }^{1}$ G. Gol'tsman, O. Okunev, G. Chulkova, A. Lipatov, A. Semenov, K. Smirnov, B. Voronov, A. Dzardanov, C. Williams, and R. Sobolewski, Appl. Phys. Lett. 79, 705 (2001).

${ }^{2}$ E. Knill, R. Laflamme, and G. Milburn, Nature (London) 409, 46 (2001).

${ }^{3}$ J. Yang, A. Kerman, E. Dauler, V. Anant, K. Rosfjord, and K. Berggren, IEEE Trans. Appl. Supercond. 17, 581 (2007).

${ }^{4}$ A. Kadin and M. Johnson, Appl. Phys. Lett. 69, 3938 (1996).

${ }^{5}$ A. Semenov, G. Gol'tsman, and A. Korneev, Physica C 351, 349 (2001).
${ }^{6}$ A. J. Kerman, E. A. Dauler, W. E. Keicher, J. K. W. Yang, K. K. Berggren, G. Gol'tsman, and B. Voronov, Appl. Phys. Lett. 88, 111116 (2006).

${ }^{7}$ D. Burnham and D. Weinberg, Phys. Rev. Lett. 25, 84 (1970).

${ }^{8}$ D. Bitauld, F. Marsili, A. Gaggero, F. Mattioli, R. Leoni, S. Nejad, F. Levy, and A. Fiore, Nano Lett. 10, 2977 (2010).

${ }^{9}$ R. Hadfield, Nat. Photonics 3, 696 (2009).

${ }^{10}$ A. Pearlman, A. Cross, W. Slysz, J. Zhang, A. Verevkin, M. Currie, A. Korneev, P. Kouminov, K. Smirnov, B. Voronov et al., IEEE Trans. Appl. Supercond. 15, 579 (2005).

${ }^{11}$ Z. Zhou, G. Frucci, S. Jahanmirinejad, F. Mattioli, A. Gaggero, R. Leoni, and A. Fiore, in Quantum Information and Measurement (Optical Society of America, 2012).

${ }^{12}$ C. Hong, Z. Ou, and L. Mandel, Phys. Rev. Lett. 59, 2044 (1987).

${ }^{13}$ G. Di Giuseppe, M. Atatüre, M. Shaw, A. Sergienko, B. Saleh, M. Teich, A. Miller, S. Nam, and J. Martinis, Phys. Rev. A 68, 063817 (2003).

${ }^{14}$ R. Byer and S. Harris, Phys. Rev. 168, 1064 (1968). 